\title{
Strategical Approach for VoLTE Performance Improvement
}

\section{Gujral $E^{*}$ and Jadon JS}

Amity Institute of Telecommunication and Mgmt., Amity University, Noida, India

\begin{abstract}
Voice over long-term evolution is stated as VoLTE. It is youngest evolution in IP technology and evolving very fast. The main drivers for VoLTE have been the fact that LTE is a packet only architecture and LTE has no inbuilt voice+SMS service engine. A solution for Voice over LTE needed as soon as mobile terminals start to use the LTE access. VoLTE provides various advantages for operator as well as end users by improving spectral and network efficiency. Deployments of VoLTE services are increasing at very fast rate and it requires number of troubleshooting and optimizations methods to improve the high definition voice quality of service. This paper presents the various aspects used to optimize the VoLTE network.
\end{abstract}

Keywords: VoLTE; KPI; Voice; Accessibility; Retainability; Mobility; Optimization

\section{Introduction}

Today, Voice over LTE (VoLTE) is the most talked about application of the entire LTE mobile network in the global telecom industry. With Voice service being the largest revenue source of all services provided in $2 \mathrm{G}$ and $3 \mathrm{G}$ systems, there was for quite some time a debate in the Industry on what should be the voice solution for LTE and different alternatives popped up threatening with fragmentation. The solution and architecture of choice should offer the benefits of Voice service evolution [1-5] while securing today's telephony service; it should support to evolve the service offering making use of broadband capabilities and easily add further revenue generating services.

There are various motivations for adopting VoLTE technology over other OTT based-VoIP services as explained in Table 1.

As VoLTE is new technology, it requires various methods to improve its voice and radio quality. In this paper we compare the OTT-VoIP and VoLTE services based on voice efficiency and quality of service. The rest of the paper is organized as: in section 2, we will discuss about related work done for optimizing VoLTE network. Section 3 presents factors to optimize the VoLTE network by comparing results with other services. Section 4 describes the performance comparison of VoLTE and other CS services. Finally conclusion has been made in section 5 .

\section{Existing Performance Improvement Solutions}

VoLTE specifications are referred as GSM Association IR.92

\begin{tabular}{|c|c|c|}
\hline \multicolumn{3}{|c|}{ VoLTE vs. OTT Services } \\
\hline Parameters & VoLTE & OTT Based VolP \\
\hline Call Setup & \multirow{2}{*}{$\begin{array}{l}\text { SIP call Control+Policy } \\
\text { control+Dedicated ERAB }\end{array}$} & \multirow{2}{*}{$\begin{array}{l}\text { Call control over IP (policy } \\
\text { control not mandatory) }\end{array}$} \\
\hline (Signaling) & & \\
\hline Signaling Load & Negligible impact & High \\
\hline $\begin{array}{l}\text { Data-packets per } \\
\text { second }\end{array}$ & $20 \mathrm{~ms}$ & Varies \\
\hline Data-bit per second & $\begin{array}{l}\text { Coding Rate+ROHC } \\
\text { overhead+IP overhead }\end{array}$ & Coding Rate+IP overhead \\
\hline Latency (Voice) & Very Less & High \\
\hline $\begin{array}{l}\text { Loaded Cell-Effort } \\
\text { (Voice) }\end{array}$ & GBR & Best \\
\hline Spectral efficiency & High & Low \\
\hline $\begin{array}{l}\text { Talk Time (Battery } \\
\text { Life) }\end{array}$ & $20 \mathrm{~ms} / 40 \mathrm{~ms} \mathrm{DRX}$ & no DRX \\
\hline $\begin{array}{l}\text { Standby Time } \\
\text { (Battery Life) }\end{array}$ & $\begin{array}{l}\text { Minimal activity at } \\
\text { background }\end{array}$ & $\begin{array}{l}\text { Frequent activities at } \\
\text { background }\end{array}$ \\
\hline
\end{tabular}

Table 1: Comparison of VoLTE vs. OTT-VoIP specifications. In 2012, early VoLTE deployment was expected and full nationwide VoLTE network by AT and T was deployed in 2013. In 2012, T. Koshimizu, I. Tanaka, and K. Nishida [6], presented idea in WTC (Wireless Communication Technical Committee) with focus on performance improvement of VoLTE and eSRVCC network using domain handover function. In 2013, Ozcan Ozturk [7], highlighted the performance of VoLTE network and suggested that Hetnet with Pico cells expansion improves the VoLTE capacity. Later, Mike Hibbered published his article that shows how VoLTE will change the ways of communication and emphasis on data handling.

In 2014, Jo, et al. [8], comes with the solution for inter-cell interference separation based resources allocation which increases the VoLTE capacity by $20 \%$. They focus on packet prioritization and frequency allocation prioritization [8]. In 2015, Sumit Gautam and Durga Parsad Sharma [9] purposed solution to reduce the voice call interruption time during mobility. Their performance target for average voice interruption time is less than $0.3 \mathrm{sec}$ and this is achieved by using ATCF (Access Transfer Control Function) and ATGW (Access Transfer Gateway) entities in network.

Currently 111 operators are investing in VoLTE in 52 countries and 30 operators (Telstra, Orange, AT and T, M1 and Viva etc.) already launched VoLTE High Definition services in 21 countries according to the report presented by GSA in October, 2015 [10-14].

Most of the previously purposed solutions [6-8] and studies focus on mobility and capacity of network. So these parameters are taken into care while designing the optimization methodology in section 3.

\section{Optimization Areas}

Deploying any technology requires various optimization and troubleshooting steps to get full advantage of technology's potential. We divided these optimization and troubleshooting areas into three major parts as given below:

*Corresponding author: Ekta Gujral, Amity Institute of Telecommunication and Management, Amity University, Noida, India. Tel: 0120244 5252; E-mail: ekta12gujral@gmail.com

Received May 12, 2015; Accepted June 23, 2016; Published July 03, 2016

Citation: Gujral E, Jadon JS (2016) Strategical Approach for VoLTE Performance Improvement. J Telecommun Syst Manage 5: 134. doi:10.4172/2167-0919.1000134

Copyright: ( 2016 Gujral E, et al. This is an open-access article distributed under the terms of the Creative Commons Attribution License, which permits unrestricted use, distribution, and reproduction in any medium, provided the original author and source are credited. 


\section{VoLTE voice quality}

- Check Discards

- Check Delays

\section{VoLTE RF quality}

- TTI Bundling Enabling

- RoHC implementation

\section{Battery saving in UE}

\section{- Efficient DRX}

In this paper we compare three voice possibilities for excellence and efficiency (Figure 1):

- Native VoLTE client: VoLTE supported smartphone.

- Non-native VoLTE clients. Application which have feature to register to IMS and establish call using QCI1.

- Over-the-Top: VoIP applications. For example Skype.

Area I: VoLTE voice quality: Voice Codec sampling rate and audio bandwidth is responsible for the voice quality. For this analysis purpose, we used narrowband bandwidth range of $80-3700 \mathrm{MHz}$ and wideband bandwidth of 50-7000 MHz. Regular call connection uses either narrowband or wideband but VoLTE by default uses wideband. Here we performed absolute category testing and mouth to ear delay testing for VoLTE, OTT and SIP services. Score is given between 1 to 10 scales i.e. is mean option score.

Figure 2 presents the scores of voice quality with different voice

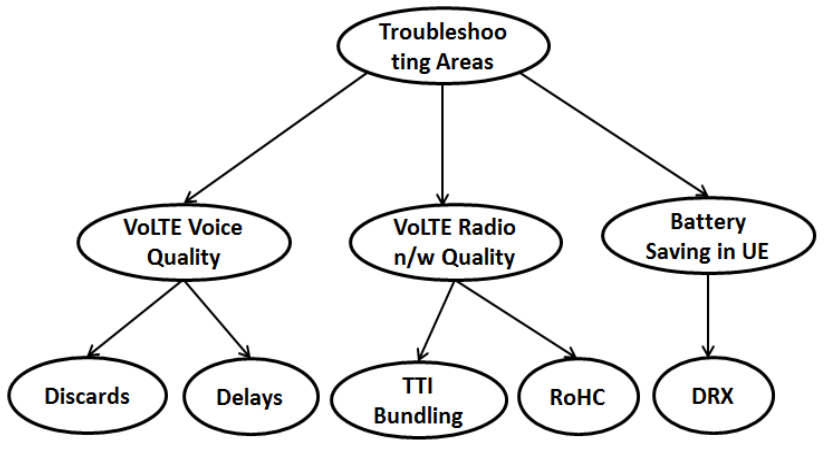

Figure 1: VoLTE troubleshooting areas.

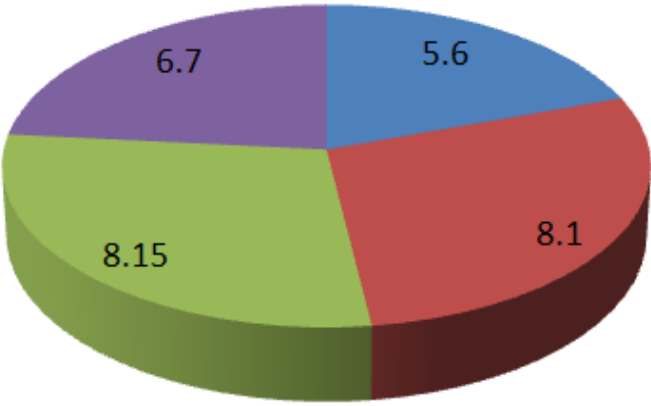

- 2G/3G Call

- VolTE Call

घ oाT Call

- SIP Call

Figure 2: VoLTE Voice Quality Results-I.

applications. VoLTE secured score of 8.1 with wideband $24 \mathrm{kbps}$. Regular 2G/3G connection scored 5.6. Other OTT application (e.g. Viber, Skype etc.) scored 8.15 and SIP calling provided 6.7. Overall OTT average score is 8.15 which are very close to VoLTE score as shown in Figure 2.

Hence we can say that voice codec helps VoLTE to match the voice quality of Over the TOP services

Now we tested the Voice quality and mouth to ear delay of OTT services and VoLTE using MAC priority scheduler [5,15] in good as well as bad RF conditions at different cell load. Cell loads percentage is measured by number of users divided by max limit of eNodeB. Considerations for test:

1. Good RF: $-75 \mathrm{dBm}$ to $-95 \mathrm{dBm}$

2. Bad RF: $-110 \mathrm{dBm}$ to $-125 \mathrm{dBm}$

3. Cell Load (\%): - $\{5,10,30,50,70,80\}$

4. Avg. score=AVG (Bad Score, Good Score)

The voice quality and mouth to ear delay for VoLTE remains constant for all loads as shown in Figure 3. Hence we can say that quality of service and smart MAC priority scheduler $[4-5,11,12]$ in the eNodeB helps to optimize the network. Secondly it helps to the paging response delay which results in reduction of call setup time of VoLTE. Average call setup time for VoLTE call in these tests is between 1.5 to 2 seconds and for other calls is nearly 3-5 seconds.

Area II: VoLTE Radio n/w quality: The Session Setup Success Rate (SSSR), Call Setup Success Rate (CSSR), Call Retainability and BLER are main key performance indicators of VoLTE. The optimizations mainly focus on these KPIs. For our paper we observed CSSR and BLER for 4 months and checked the results with following considerations:

1. TTI bundling: Feature disable on eNodeB

2. TTI bundling: Feature enable on eNodeB

3. RoHC- Feature disable on eNodeB

4. RoHC-Feature enable on eNodeB

With enabling the TTI bundling, uplink become more robust and increases the coverage by 5-6 dB. During RF bad conditions BLER and CSSR reduced without TTI bundling as shown in Figure 4:

Similarly, we experiment eNodeB with and without robust header compression feature. Robust header compression basically runs between UE and eNodeB. In small chunks of data RoHC helps to increase the network capacity. For example if the actual data size is 20

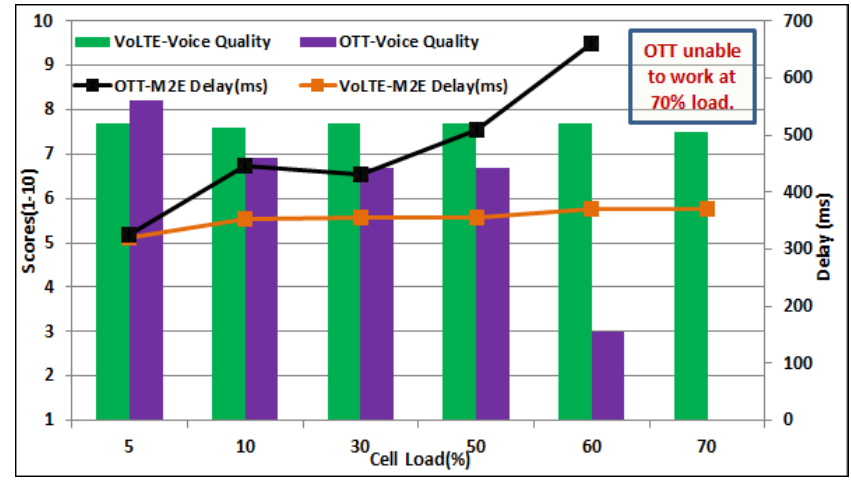

Figure 3: VoLTE Voice Quality Results-II. 


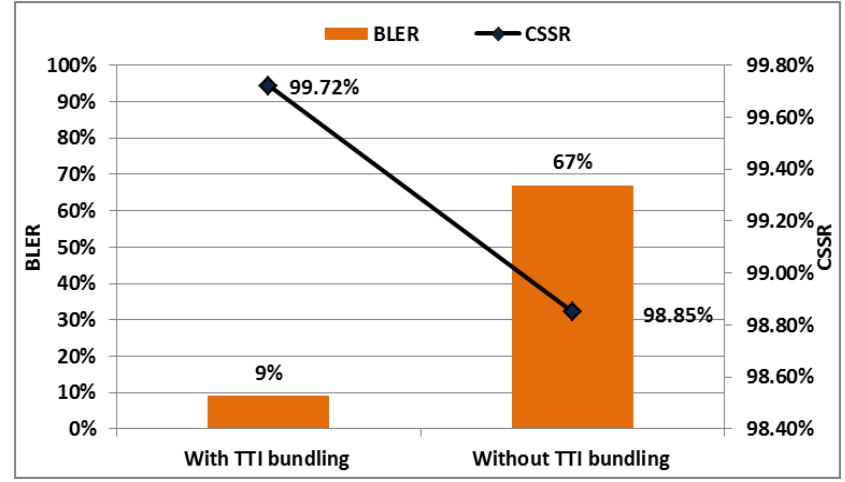

Figure 4: VoLTE RF Quality Results.

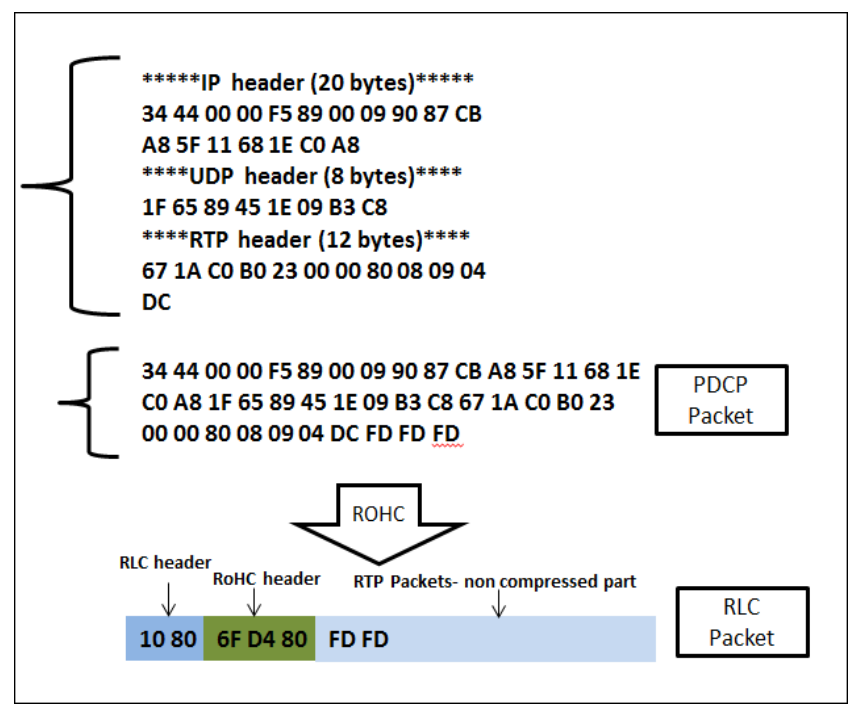

Figure 5: VoLTE RoHC compression.

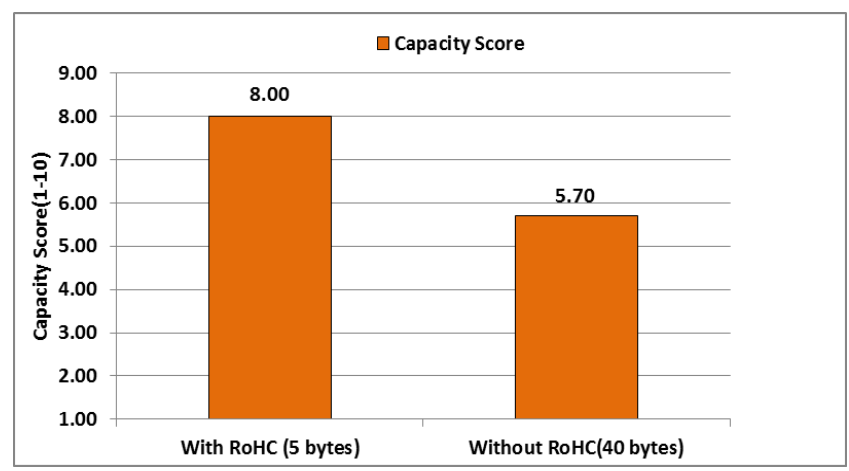

Figure 6: Benefits of RoHC.

bytes and IP overhead [12-14] is 60 bytes, then most of the resources are blocked by header than the data. So in such scenario RoHC feature plays important role and increases capacity. In VoLTE, SIP and Voice traffic packets are two main kinds of packets. For SIP signaling part, RoHC does not apply on SIP signaling [12-14] and only applicable for data packets because for SIP signaling packet size is relatively large as compared to header size. RoHC functionality describes in Figure 5.

We tested capacity of eNodeB with 40 Bytes IP header (without RoHC) and with 5 bytes IP header (with RoHC). Capacity of network increased by $5 \%$ with RoHC and helps to admit more calls at given time. Figure 6 shows the score card of capacity with and without RoHC feature in eNodeB.

Area III: Battery saving in UE: Power consumption of phone is very important factor for smartphone users. UE battery can extend either by handset architecture modification or by efficient DRX feature in network. DRX is discontinuous reception, it used sleep mode in handset during reception of packets (every $20 \mathrm{~ms}$ ). The DRX can be activated in such a way that two voice packets are able to transmit simultaneously and it increases the packet arrival time by $30-40 \mathrm{~ms}$ [16]. During our analysis we found that with DRX activation device power consumption reduced by $30-40 \%$ as shown in Figure 7 [17].

\section{Performance Comparison}

Score card for all optimization scenario are given below for voice quality. It shows that with smart MAC priority scheduler efficiency and capacity (10-20\%) of VoLTE network can be enhanced (Table 2).

Table 3 measurements shows that with the help of TTI bundling and RoHC feature, we can control accessibility and interference of network and results in strong key performance indicators of network. Table 4 shows that by using efficient DRX in network, we can easily enhance the battery life by $30-40 \%$.

\section{Conclusion}

VoLTE is growing technology and supported by various new VoLTE capable devices. It enhances the operator's overall OPEX, CAPEX, and spectral efficiency and also provides high quality of

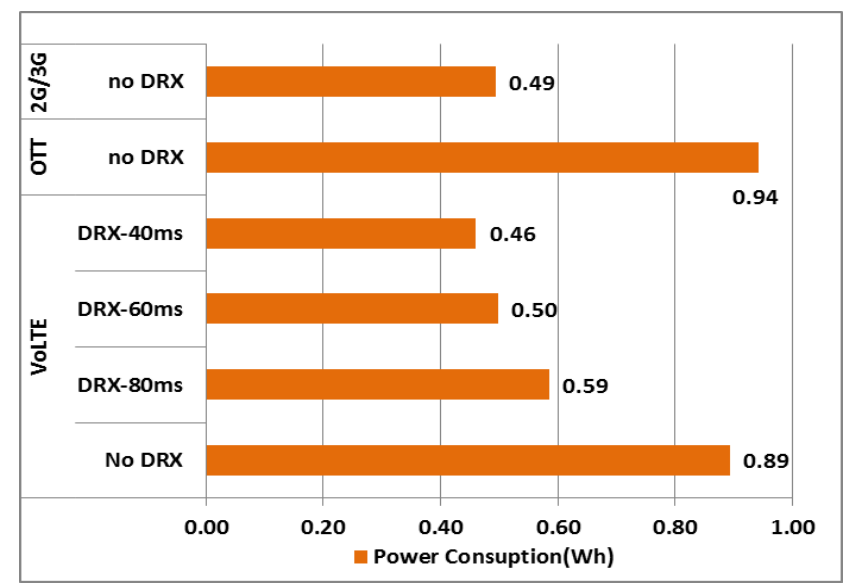

Figure 7: Benefits of DRX activation.

\begin{tabular}{|l|c|c|c|}
\hline Voice Quality \\
\hline Parameters & VoLTE & SIP & OIT \\
\hline Different voice applications (1-10) & 8.1 & 6.7 & 8.15 \\
\hline Voice Quality (1-10) & 7.65 & & 5.25 \\
\hline M2E Delay(ms) & 353 & & 474 \\
\hline
\end{tabular}

Table 2: VoLTE Voice Quality Optimization Results.

\begin{tabular}{|l|l|l|}
\hline Radio Quality & With TTI/RoHC & Without TTI/RoHC \\
\hline Parameters & 99.7 & 98.85 \\
\hline CSSR (\%) & 9 & 67 \\
\hline BLER (\%) & 8 & 5.7 \\
\hline Cap. Score(1-10) & 8 & \\
\hline
\end{tabular}

Table 3: VoLTE Radio Quality Optimization Results. 
Citation: Gujral E, Jadon JS (2016) Strategical Approach for VoLTE Performance Improvement. J Telecommun Syst Manage 5: 134. doi:10.4172/21670919.1000134

\begin{tabular}{|l|c|c|c|}
\hline Battery Life \\
\hline Parameters & VoLTE & $2 \mathrm{G} / 3 \mathrm{G}$ & OIT \\
\hline Power Consumption(Wh) & 0.51 & 0.49 & 0.94 \\
\hline
\end{tabular}

Table 4: Battery Life- Efficient DRX

service at end user. This paper demonstrates the various aspects to optimize the voice quality, radio network quality and methods to save UE battery life. It also informs about the related features required to optimize network. It is observed that VoLTE provides higher speech/voice quality, excellent network performance even in poor RF conditions and low power consumption over the OTT services. Hence network reliability can be achieved by activating various VoLTE related QoS features in network.

\section{References}

1. Policy and Charging Control Architecture 3GPP (2013)

2. Quality of Service concept and architecture 3GPP (2014).

3. 3GPP: IP Multimedia Subsystem (IMS) Stage 2.TS23.228 (2014).

4. 3GPP: Medium Access Control (MAC) protocol specification. TS36.321 (2015).

5. Transport Protocol for Real-Time Applications. RFC 3550 (2003).
6. Tanaka I, Nishida K, Koshimizu T (2012) Improvement on the Voice over LTE Handover with Operator's Vision. IEEE Xplore.

7. Vajapeyam M, Ozturk O (2013) Performance of VoLTE and data traffic in LTE heterogeneous networks. IEEE GLOBECOM.

8. Byungkab Jo, Park T, Lee W, Ryu B, Choi S (2014) Inter-cell interference separation-based resource allocation for VoLTE. IEEE GLOBECOM.

9. Gautam S, Sharma DP (2015) Solution to Reduce Voice Interruption Time during Handover of VoLTE. 5th International Conference on Advanced Computing and Communication Technologies.

10. GSA Evolution to LTE report- VoLTE global status.

11. IR.92 IMS Profile for Voice and SMS V3.0.GSMA (2010)

12. IR.94 IMS Profile for Conversational Video Service V10.0 (2011).

13. 3GPP: Single Radio Voice Call Continuity (SRVCC) TS.23.216 (2011).

14. IR.64 IMS Service Centralization V2.0 (2012).

15. GSMA-VoLTE.

16. VoLTE optimization: Nokia volte optimization white paper (2013).

17. 3GPP: Service Requirements for the Evolved Packet System (EPS). TS22.278 (2011). 